\title{
Comparison of Compact Reduced Basis Method with Different Model Order Reduction Techniques
}

\author{
Damian Szypulski , Grzegorz Fotyga , Valentín de la Rubia and Michal Mrozowski
}

\begin{abstract}
Different strategies suitable to compare the performance of different model order reduction techniques for fast frequency sweep in finite element analysis in Electromagnetics are proposed and studied in this work. A Frobenius norm error measure is used to describe how good job a reduced-order model is doing with respect to the true system response. In addition, the transfer function correct behavior is monitored by studying the convergence of the poles and zeros of the reduced-order system. The results of this comparison are detailed in two microwave filter structures.
\end{abstract}

Index Terms-Computer aided engineering, error analysis, finite element methods, Galerkin method, reduced basis methods, reduced order systems.

\section{INTRODUCTION}

Model-order reduction (MORe) became an indispensable tool to perform an efficient frequency-domain analysis in the area of computational electromagnetics (CEM), by means of the finite element (FE) as well as finite difference (FD) techniques. The main idea of MORe is to properly construct a set of orthogonal vectors, which span a low-dimensional subspace, in which an approximate solution to the original system of equations is sought.

One of the most commonly used reduction techniques in CEM is called the reduced basis method (RBM) [1], [2], [3], [4]. In this technique, the projection basis is composed of solution snapshots, which are properly selected using a greedy algorithm. More precisely, the subsequent snapshot is computed at the frequency in which the efficiently computed a-posteriori error estimator indicates the largest error. The drawback of RBM is that it needs a relatively large number of snapshots to generate a high-accuracy reduced-order model (ROM) for the wide-frequency band analysis, and each snapshot requires a rather time-consuming FEM system matrix factorization.

A different strategy has been proposed in the technique called the subspace accelerated dominant pole algorithm SADPA [5] and in [6], where the projection space is composed of the eigenmodes, to approximate the transfer function of the original system.

Recently we proposed a new reliable model-order reduction approach for fast frequency sweep [7], which relies on RBM and resonant mode expansion methods. Namely, it generates the orthogonal projection basis that is composed of two groups of vectors:

- eigenvectors of the FEM system of equations, which are associated with the frequencies from the analyzed frequency bandwidth,

- solution snapshots computed at properly selected frequency points.

The numerical tests provided in [7] have proved that the proposed method significantly increases the efficiency of the frequency domain FEM analysis, both in the narrow and the wide-band simulations.

The aim of this paper is to provide a deeper insight into the quality of the ROM generated in the proposed methodology in [7], in comparison with standard RBM [4] and proper orthogonal decomposition (POD) [1].

\section{PRoblem Statement}

Let us consider a two-port microwave circuit, namely, a microwave filter. The filter transfer function is represented by an impedance matrix transfer function. After discretization of the time-harmonic Maxwell's equations by means of FEM, we get the following linear dynamical system representation of the microwave circuit:

$$
\left(\begin{array}{c}
v_{1} \\
v_{2} \\
0
\end{array}\right)=\left(\begin{array}{ccc}
0 & 0 & j b_{1}^{T} \\
0 & 0 & j b_{2}^{T} \\
s j b_{1} & s j b_{2} & S+s^{2} T
\end{array}\right)\left(\begin{array}{c}
i_{1} \\
i_{2} \\
x
\end{array}\right),
$$

where $S$ and $T$ are the stiffness and mass matrices, $x$ stands for the state-space variable and $s$ is the complex frequency. Once the state-space variable, which represents the FEM discretization of the electric field, is eliminated from equation (1), the impedance matrix transfer function arises, thus,

$$
\left(\begin{array}{l}
v_{1} \\
v_{2}
\end{array}\right)=-s\left(\begin{array}{l}
j b_{1}^{T} \\
j b_{2}^{T}
\end{array}\right)\left(S+s^{2} T\right)^{-1}\left(\begin{array}{ll}
j b_{1} & j b_{2}
\end{array}\right)\left(\begin{array}{l}
i_{1} \\
i_{2}
\end{array}\right) .
$$

It should be noted that the dynamical system (1) has a rather large state-space, thus solving this system frequency by frequency cannot be afforded. In this regard, we use MORe to carry out a fast frequency sweep. The goal in MORe is to identify the manifold, or reduced-basis space, in which the actual dynamics of system (1) take place in an efficient way. 


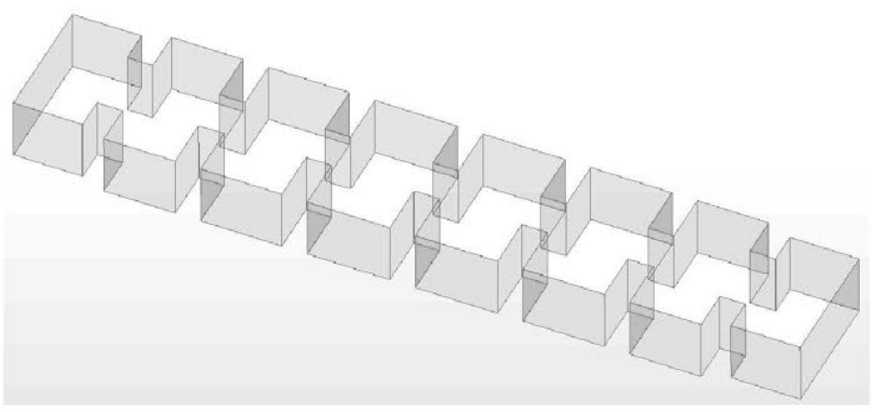

Fig. 1. 6th-order H-plane filter example.

The purpose in this work is to carry out a fair comparison among different MORe techniques in order to be able to identify the best performances. The key is to find the right measure to describe the benefits in MORe.

\section{ROM PERFORMANCE MEASURE}

First, one needs the condition to measure the quality of the reduced-basis space used to approximate the state-space variable in the dynamical system (1). Let us take into account a collection of solution snapshots for the electric field (statespace variable) in (1) throughout a specific frequency band, and put them altogether in a matrix, namely, $A$ matrix. In the same token, let us take into account the same collection of solution snapshots for the electric field but this time obtained via MORe for the same frequency band of interest and, once again, put them altogether in a matrix, namely, $A_{M O R e}$ matrix. The Frobenius norm of the difference matrix $A-A_{M O R e}$ indicates how good job the ROM is doing in order to properly describe the original dynamical system (1). As a result, we obtain a good measure for the performance of the ROM.

Second, we can take into account how good the system transfer function response (2) is described by the ROM. In this sense, since the transfer function (2) is indeed a rational function, we may consider the convergence in the poles and zeros in the rational function as a MORe performance measure criterion. This kind of convergence has to be achieved in order to get a reliable ROM, otherwise the system transfer function characteristics will not be properly addressed.

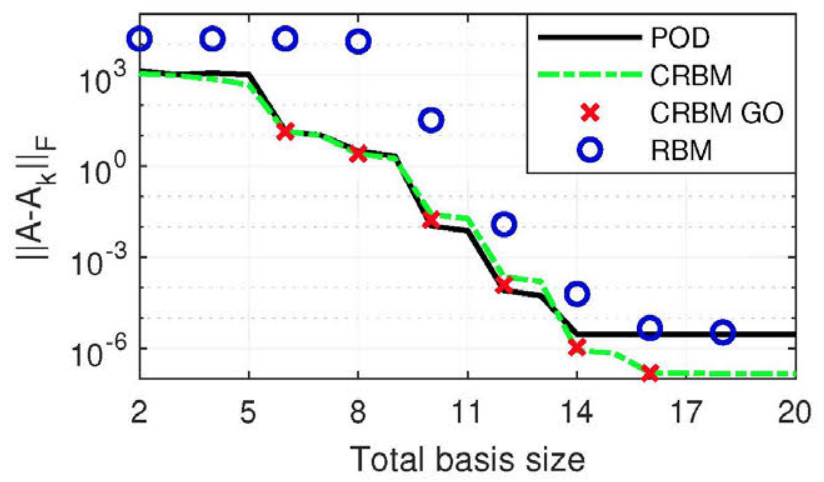

Fig. 2. H-plane filter example. Frobenius norm error convergence.

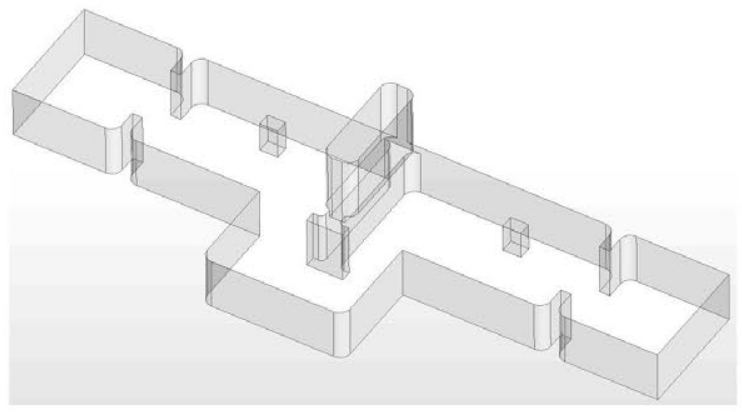

Fig. 3. 5th-order waveguide filter example.

Finally, the last criterion to measure the accuracy in MORe is the input-output error in terms of S parameters description of the microwave circuit.

In this work, two standard approaches have been taken into account which are based on solution snapshots, namely, POD and RBM. POD gives information about the best combination among a set of solution vectors within a specific frequency band resulting in the best and the smallest reduced-basis space. However it requires to carry out a singular value decomposition procedure (SVD), which is time and memory consuming, since it takes into account a full set of solution vectors, which is practically not applicable for single shot simulations. The RBM algorithm is based on error estimator and this approach efficiency is relatively close to POD in terms of basis size with a specific error tolerance.

\section{NUMERICAL RESULtS}

In this section, we compare the Compact Reduced Basis Method (CRBM) detailed in [7] under two different flavors to RBM and POD approaches. The first CRBM flavor is detailed in [7] where each additional snapshot needed in the reducedbasis space is chosen based on a linearly independent criterion, whereas the second CRBM flavor takes into account a goaloriented (GO) error estimator as snapshot selection criterion. In both CRBM flavors, we start off from a fundamental basis composed of eigenresonances obtained via the solution of generalized eigenproblem. Only eigenresonances that hit the band are taken into account. Starting with this basis we

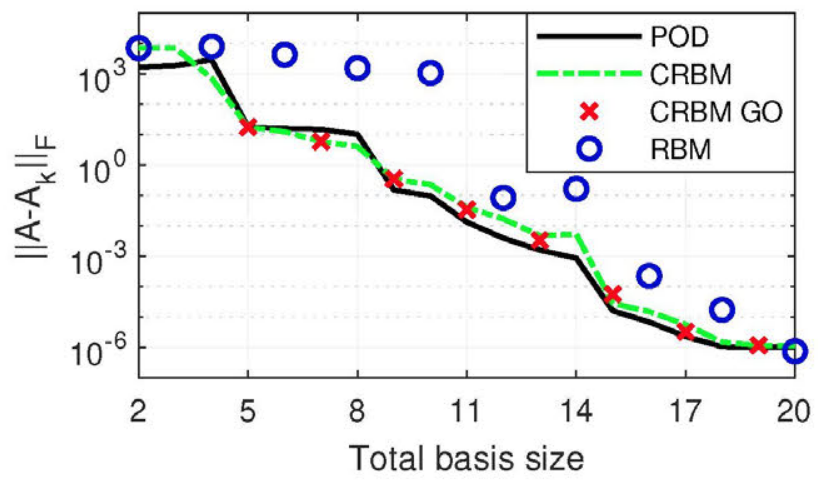

Fig. 4. 5th-order waveguide filter example. Frobenius norm error convergence. 


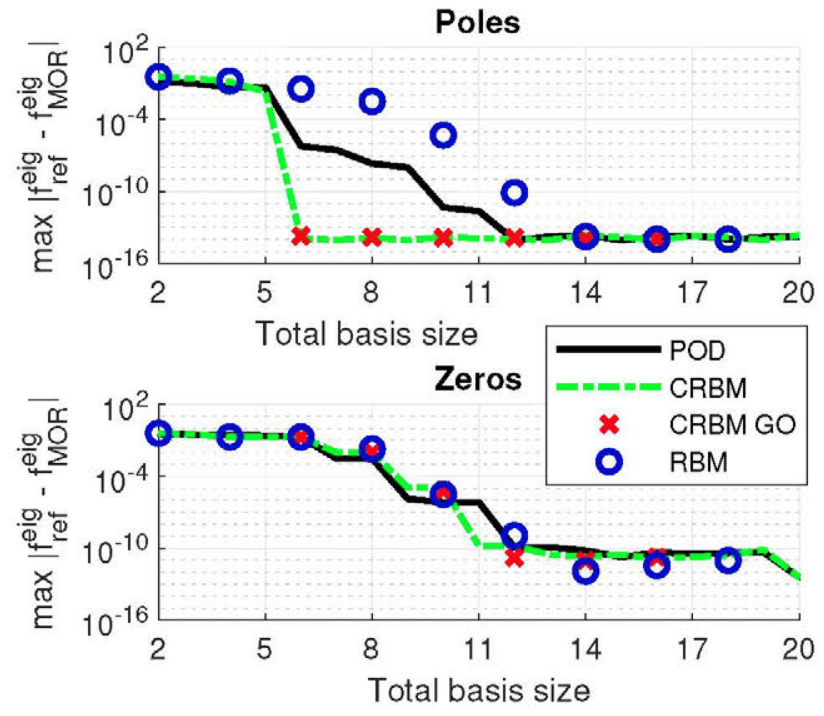

Fig. 5. H-plane filter example. Pole and zero error convergence.

continue selecting subsequent snapshots by using either a linear independence measure or a goal-oriented error estimator until a given tolerance is achieved.

The first set of results refers to a 6th-order filter depicted in Fig. 1. This is a filter based on the WR-75 waveguide and is analyzed in $12.5-13.5 \mathrm{GHz}$ band. There are 6 eigenfrequencies that hit in the frequency band of interest. In Fig. 5 , the maximum frequency difference between poles and zeros have been shown. CRBM poles have already converged due to eigenvectors included to the basis in the first step. The Frobenius norm is strongly correlated to POD and as well as the maximum $S$ parameter error which are shown in Fig. 2 and Fig. 6.

Our second example is a 5th-order waveguide filter [8] detailed in Fig. 3. We carry out a model-order reduction in the 9.2-10.8 GHz band in order to obtain a fast frequency sweep in the filter response. Since a fifth-order filter is designed, 5 eigenvectors are found in the filter operation frequency band. The maximum frequency difference between poles and zeros is shown in Fig. 7. Once again, CRBM poles have already converged due to eigenvectors included to the basis in the

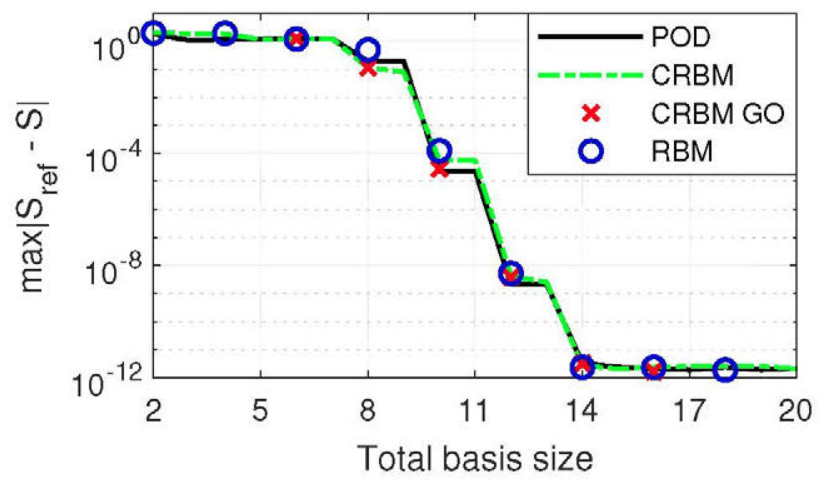

Fig. 6. H-plane filter example. S parameter error convergence.

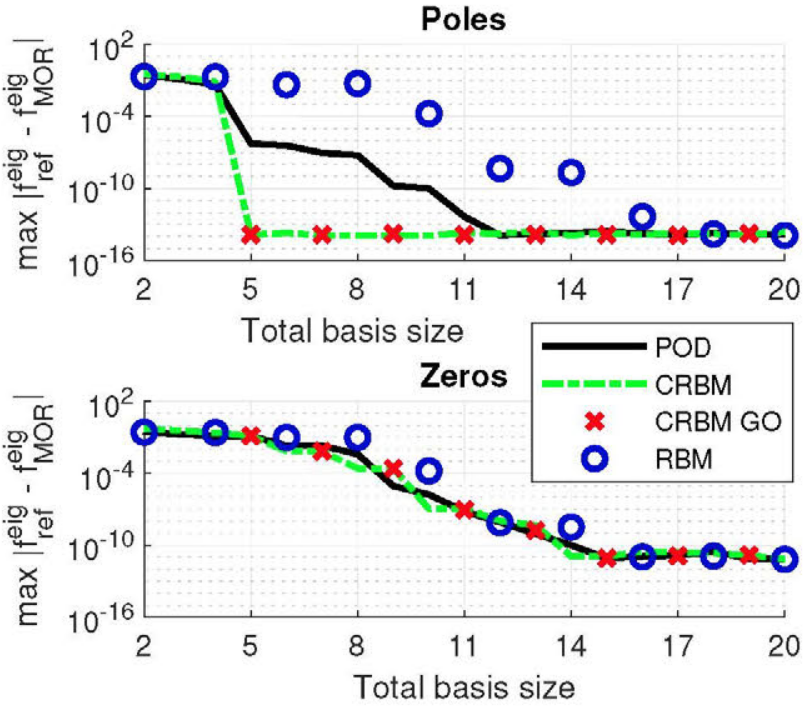

Fig. 7. 5th-order waveguide filter example. Pole and zero error convergence.

first step. Frobenius norm results detailed in Fig. 4 show that POD and CRBM are behaving in a very similar way in terms of performance. Maximum $S$ parameter error performance in shown in Fig. 8.

\section{CONCLUSION}

Different strategies have been studied to compare the performance of different model order reduction techniques for fast frequency sweep in finite element analysis in electromagnetics. A deeper understanding in the performance of the ROMs generated in CRBM, RBM and POD have been possible due to the introduction of Frobenius norm error measure and polezero error convergence from the circuit characteristics of the system under analysis. The numerical results, which deal with the two waveguide filters, show that CRBM and POD are behaving in a very similar way in terms of the quality of the reduced-basis space, and the convergence of the poles and zeros of the reduced-order system.

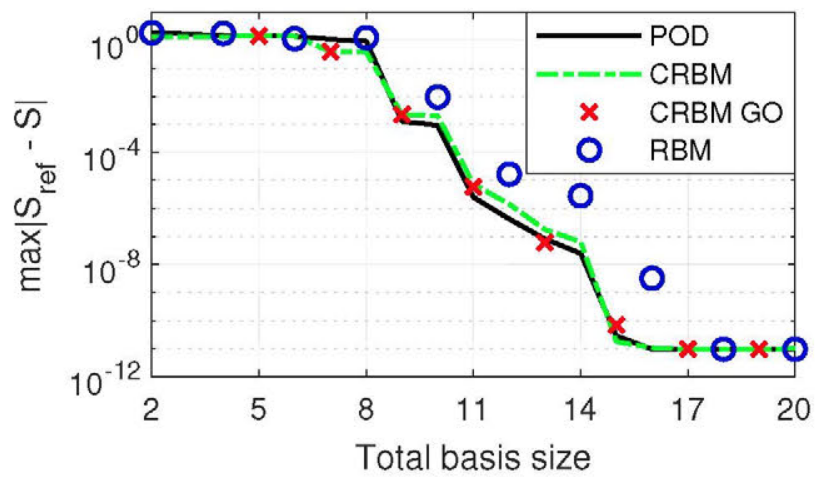

Fig. 8. 5th-order waveguide filter example. $\mathrm{S}$ parameter error convergence. 


\section{ACKNOWLEGDEMENTS}

This work was supported by the Foundation for Polish Science within the TEAM-TECH program, cofinanced by the European Regional Development Fund, Smart Growth Operational Program 2014-2020 (project EDISOn: Electromagnetic Design of flexIble SensOrs).

\section{REFERENCES}

[1] A. Quarteroni, A. Manzoni, and F. Negri, Reduced Basis Methods for Partial Differential Equations: An Introduction. Springer, 2016, vol. 92.

[2] V. de la Rubia, U. Razafison, and Y. Maday, "Reliable fast frequency sweep for microwave devices via the reduced-basis method," IEEE Trans. Microw. Theory Techn., vol. 57, no. 12, pp. 2923-2937, Dec. 2009.

[3] M. Rewienski, A. Lamecki, and M. Mrozowski, "A goal-oriented error estimator for reduced basis method modeling of microwave devices," IEEE Microw. Compon. Lett., vol. 25, no. 4, pp. 208-210, Apr. 2015.

[4] V. de la Rubia, "Reliable reduced-order model for fast frequency sweep in microwave circuits," Electromagnetics, vol. 34, no. 3-4, pp. 161-170, 2014.

[5] J. Rommes and N. Martins, "Efficient computation of transfer function dominant poles using subspace acceleration," IEEE Transactions on Power Systems, vol. 21, no. 3, pp. 1218-1226, 2006.

[6] F. D. Freitas, J. Rommes, and N. Martins, "Gramian-based reduction method applied to large sparse power system descriptor models," IEEE Transactions on Power Systems, vol. 23, no. 3, pp. 1258-1270, 2008.

[7] V. de la Rubia and M. Mrozowski, "A Compact Basis for Reliable Fast Frequency Sweep via the Reduced-Basis Method," IEEE Transactions on Microwave Theory and Techniques, vol, 66, no. 10, pp. 4367-4382, 2018.

[8] L. Szydlowski and M. Mrozowski, "A self-equalized waveguide filter with frequency-dependent (resonant) couplings," IEEE Microwave Wireless Compon. Lett., vol. 24, no. 11, pp. 769-771, Nov. 2014. 